
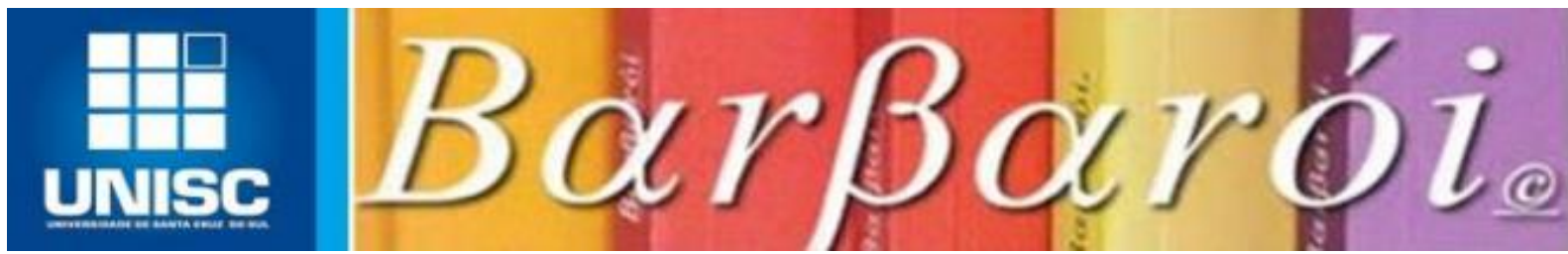

\title{
O SERVIÇO SOCIAL NO ATENDIMENTO DE EMERGÊNCIAS PSIQUIÁTRICAS: PROCESSOS DE TRABALHO DE ASSISTENTES SOCIAIS E RESIDENTES NO ATENDIMENTO DE PACIENTES ADOLESCENTES COM IDEAÇÃO E TENTATIVA DE SUÍCIDIO
}

\author{
DOI: http://dx.doi.org/10.17058/barbaroi.v51i1.12913 \\ Carlos Stavizki Junior \\ Universidade de Santa Cruz do Sul-UNISC - Brasil \\ Eunice Maria Viccari \\ Universidade de Santa Cruz do Sul-UNISC - Brasil
}

\section{RESUMO}

Este trabalho apresenta reflexões sobre o fenômeno do suicídio na adolescência, a partir da análise dos processos de trabalho de Assistentes Sociais residentes de um Serviço de Emergência do SUS. Este estudo surge pela necessidade de qualificar metodologias de trabalho condizentes com os princípios profissionais e éticos do Serviço Social e Saúde Mental. Trata-se de um estudo descritivo com abordagem qualitativa, constituído por seis Assistentes Sociais, sendo três ex-residentes do Programa de Residência Multiprofissional em Saúde com ênfase em Intensivismo, Urgência e Emergência e três Assistentes Sociais do Hospital Santa Cruz. A coleta de dados foi realizada através de entrevista semiestruturada entre os meses de março e junho de 2018. Descobriu-se que 83\% das profissionais realizaram atendimentos no Serviço de Emergência e 67\% realizaram intervenções diretas com adolescentes com tentativa ou ideação suicida. Desvendou-se o fluxo de atendimento destes casos como o acolhimento, planejamento, encaminhamentos e o monitoramento do paciente, atribuindo a qualificação do atendimento ao aprimoramento profissional decorrente do Programa de Residência Multiprofissional em Saúde.

Palavras-chave: Suicídio; Serviço Social; Adolescência; Serviço de Emergência.

\section{INTRODUÇÃO}

Nas últimas décadas, muitos estudos e dados epidemiológicos apontam para o aumento exponencial dos casos de morte por suicídio no Brasil, assim como a nível mundial, principalmente na faixa etária entre 15 e 19 anos, sendo considerada a terceira causa de morte entre os adolescentes (BERTOLOTE \& FLEISCHMANN, 2004; DE LEO, BERTOLOTE, \& LESTER, 2003). A adolescência é um período da vida marcado por diversas mudanças e contradições entre o indivíduo, as pessoas que fazem parte do seu convívio e o mundo que lhe cerca. $\mathrm{O}$ estresse causado pelas situações de conflito que essas mudanças exigem coloca o 
adolescente, muitas vezes, em vivências de isolamento, solidão, desesperança e sofrimento. A literatura apresenta que "dos adolescentes que tentaram suicídio, 10\% acabam se suicidando dentro de 10 anos após a primeira tentativa e que somente $25 \%$ dos que tentaram procuraram consulta médica, pois acreditam que podem resolver seus problemas sem ajuda" (AVANCI, PEDRÃO \& COSTA JÚNIOR, 2005, pg.: 536).

O suicídio de adolescentes já é considerado uma questão de saúde pública, necessitando de adequação e aprimoramento dos serviços de saúde para atuar nesta problemática. No Brasil, algumas medidas estão sendo tomadas, como a criação e implementação da Rede de Atenção Psicossocial (RAPS), pela Portaria n ${ }^{\circ}$ 3088/GM/MS, de 23 de dezembro de 2011. Os objetivos da RAPS são: Ampliar o acesso à atenção psicossocial da população em geral; promover a vinculação das pessoas com transtornos mentais e com abuso do uso de crack, álcool e outras drogas e suas famílias; e garantir a articulação e integração dos pontos de atenção das redes de saúde no território, qualificando a assistência por meio do acolhimento, do acompanhamento contínuo e da atenção às urgências (BRASIL, 2011).

Para atender as urgências em Saúde Mental, os Serviços de Emergência tiveram que adequar-se à nova legislação, instituindo equipes capacitadas a atender os mais complexos casos psiquiátricos, além de profissionais especializados no manejo destes pacientes. $\mathrm{O}$ Serviço de Emergência (SE) do Hospital Santa Cruz, conta, desde 2011, com o suporte técnico do Serviço Integrado de Atendimento Psicossocial (SIAP), composto por Assistentes Sociais e Psicólogos, as quais atuam como equipe de apoio para casos de ideação e tentativa de suicídio, entre outras. Em 2014, com a formação da primeira turma do Programa de Residência Multiprofissional em Saúde $(\mathrm{PRMS})^{1}$ com ênfase em Urgência, Emergência e Intensivismo, pode-se qualificar as intervenções aos pacientes de Saúde Mental. A partir do programa, pode-se incluir à equipe de urgência e emergência do Hospital, um Assistente Social de referência, sendo o residente de segundo nível (R2) de Serviço Social do PRMS. Desde então, a qualificação e aperfeiçoamento dos procedimentos de trabalho vem sendo tarefa cotidiana dos profissionais que atuam com esta expressão da questão social - o suicídio.

Este trabalho apresentará a análise de dados coletados com as Assistentes Sociais que

\footnotetext{
${ }^{1}$ O Hospital Santa Cruz (HSC) foi contemplado, através do Edital n o 32, de 24 de junho de 2014, com a implantação do Programa de Residência Multiprofissional em Saúde (PRMS) oferecendo anualmente, através do Ministério da Saúde, 20 bolsas distribuídas nas áreas de Nutrição (duas), Fisioterapia (quatro), Enfermagem (quatro), Farmácia (quatro), Psicologia (duas), Odontologia (duas), Educação Física (uma) e Serviço Social (uma). A duração do programa é de dois anos, sendo o mesmo desenvolvido nas dependências do Hospital, da Universidade de Santa Cruz do Sul (Unisc) e da Secretaria Municipal de Saúde de Santa Cruz do Sul. A área de concentração de formação dos Residentes é a de Especialista em Intensivismo, Urgência e Emergência configurando em ensino de pós-graduação Lato Sensu.
} 
participaram do PRMS e as profissionais de Serviço Social que atuaram na instituição nos últimos cinco anos, visando identificar os processos de trabalho destas profissionais diante destes casos. O objetivo é analisar os procedimentos e instrumentais de trabalho utilizados pelo Serviço Social em um Serviço de Emergência, especialmente no atendimento a adolescentes que planejam ou tentaram o suicídio. Será avaliada ainda a relação entre as diferentes áreas da saúde e a relevância da formação ofertada pelo PRMS para a qualificação do atendimento a estes pacientes.

Ao final, será discutido sobre as possibilidades de intervenção para os Assistentes Sociais atuantes em unidades de emergência, visando contribuir para o debate profissional. A atuação do Serviço Social na saúde tem seu caminho marcado pela necessidade de consolidação de seu projeto ético-político, sendo necessário instigar e qualificar constantemente este debate. Veremos que atuar em serviços de saúde onde exista a necessidade de respostas ágeis e resolutivas, diante de demandas graves e complexas como a questão do suicídio, é um desafio não apenas para os Assistentes Sociais, mas para todos os profissionais da saúde. Por fim, apresentaremos as considerações das profissionais entrevistadas sobre a formação de especialista em Urgência, Emergência e Intensivismo. 


\section{OBJETIVO}

Analisar os processos de trabalho dos Assistentes Sociais e residentes do Programa de Residência Multiprofissional em Saúde do Hospital Santa Cruz, com ênfase em Urgência, Emergência e Terapia Intensiva, em casos de tentativa de suicídio ou ideação suicida de adolescentes atendidos no Serviço de Emergência.

\section{MÉTODO}

\section{População}

A população total inclusa no presente estudo foi de seis Assistentes Sociais, distribuídas da seguinte forma: 06 (100\%) do sexo feminino; 03 (50\%) ex-residentes do Programa de Residência Multiprofissional do Hospital Santa Cruz; 02 (33,3\%) funcionárias do Serviço Integrado de Atendimento Psicossocial do Hospital Santa Cruz; 01 (16\%) extrabalhadora do Hospital Santa Cruz. A população da pesquisa representa $100 \%$ das trabalhadoras que exerceram a função de Assistente Social do Hospital Santa Cruz nos últimos cinco anos.

\section{Procedimento}

Os dados foram obtidos através de estudo descritivo e reflexivo, com abordagem qualitativa e coleta de dados através de entrevista semiestruturada, realizada entre março e junho de 2018, seguindo o método exposto por Minayo (2003). O formulário de entrevista baseou-se em eixos norteadores, sendo: (1) Atribuições do assistente social em Serviços de Emergência; (2) Procedimentos de trabalho do Serviço Social a pacientes adolescentes; (3) Processos de trabalho do assistente social em casos de Saúde Mental; (4) Encaminhamentos realizados pela equipe multiprofissional de saúde do Serviço de Emergência do Hospital Santa Cruz em casos de tentativa de suicídio de adolescentes; e (5) Comunicação entre as equipes de saúde do Hospital Santa Cruz e Rede de Saúde Mental de Santa Cruz do Sul. As variáveis foram o efetivo atendimento aos adolescentes com tentativa de suicídio no Serviço de Emergência e fazer parte do PRMS. A pesquisa foi submetida aos trâmites legais necessários, sendo aprovada pelo Comitê de Ética da Universidade de Santa Cruz do Sul UNISC. Parecer CEP n ${ }^{\circ}$ 2.445.499, CAAE: 80813817.6.0000.5343. 


\title{
RESULTADOS
}

\section{O trabalho do Assistente Social em Serviços de Emergência}

A atuação profissional do Assistente Social - considerando suas atribuições e competências, seja na saúde ou nos demais espaços sócio-ocupacionais - é norteada pelos direitos e deveres firmados no Código de Ética Profissional (Lei no 8.662/93). No âmbito da saúde o Assistente Social desenvolve seu trabalho no processo de mediação entre as necessidades e direitos dos pacientes para garantia de sua saúde, no acesso aos bens e serviços necessários para promoção e reestabelecimento da saúde, além da comunicação com outras Políticas Públicas Sociais.

O Assistente Social possui o instrumental teórico-prático necessário para realizar intervenções adequadas à realidade, buscando proporcionar às famílias o acesso a direitos, e às crianças e adolescentes, uma efetiva proteção.

\begin{abstract}
Nos vários espaços de atuação, os assistentes sociais trabalham com a questão social nas suas mais variadas expressões cotidianas, tais como os indivíduos a experimentam no trabalho, na família, na área habitacional, na saúde, na assistência social pública etc. Questão social que, sendo desigualdade é também rebeldia, por envolver sujeitos que vivenciam as dificuldades e a ela resiste e se opõem. (IAMAMOTO, 2007, p. 28).
\end{abstract}

Os profissionais devem possibilitar o acesso a direitos, à informação, à participação efetiva dos sujeitos, bem como o fortalecimento e articulação entre seus usuários e a rede socioassistencial e de saúde. É necessário romper com o caráter assistencialista e objetivos imediatistas da sociedade, capacitando os indivíduos atendidos, a fim de que sejam capazes de acessar seus direitos sociais. O trabalho deve respeitar e se orientar pelos princípios e as leis ${ }^{2}$ que norteiam o trabalho do Assistente Social, o qual deve assumir uma postura ética e reflexiva ao seu próprio processo de trabalho. A atuação deste profissional em Serviços de Emergência exercita diariamente esses conceitos, seja pela articulação com outras áreas da saúde e suas diferentes práticas profissionais, pela característica imediatista ou pela falta de formação específica para atuação em Serviços de Emergência.

Este estudo verificou que, em relação à população pesquisada, $05(83 \%)$ realizaram atendimentos no Serviço de Emergência; 04 (67\%) realizaram intervenções diretas com adolescentes com tentativa ou ideação suicida; e 03 (50\%) realizaram estágio supervisionado no Centro de Atenção Psicossocial da Infância e Adolescência de Santa Cruz do Sul (CAPSIA). O quadro a seguir mostra os resultados obtidos durante a pesquisa.

\footnotetext{
${ }^{2}$ A profissão de Assistente Social baseia-se em valores éticos, fundamentados na definição mais abrangente, de compromisso com os usuários, com base na liberdade, democracia, cidadania, justiça e igualdade social, expressos no Código de Ética do/a Assistente Social e na Lei no 8.662 de 1993.
} 
Quadro 1. Distribuição dos Procedimentos de Trabalho do Serviço Social em casos de Saúde Mental no Serviço de Emergência, elencados pelos profissionais entrevistados.

\begin{tabular}{|l|l|l|}
\hline Passos & $\begin{array}{l}\text { Processos de Trabalho do/a } \\
\text { Assistente Social }\end{array}$ & Descrição \\
\hline Passo 01 & Acolhimento/ Atendimento & $\begin{array}{l}\text { Acolhimento ao paciente e familiares ou } \\
\text { responsáveis, visando identificar o contexto sócio- } \\
\text { familiar, escolar e de saúde do paciente. }\end{array}$ \\
\hline Passo 02 & Levantamento de informações & $\begin{array}{l}\text { Contato com serviços da Rede de Saúde Mental do } \\
\text { município, ESF e de Assistência Social para } \\
\text { verificar acompanhamento ao núcleo familiar ou } \\
\text { incidências de episódios psiquiátricos do paciente. }\end{array}$ \\
\hline Passo 03 & Planejamento & $\begin{array}{l}\text { Discussão dos casos com profissional de } \\
\text { psicologia e equipe médica do Serviço de } \\
\text { Emergência. }\end{array}$ \\
\hline Passo 04 & Encaminhamentos & $\begin{array}{l}\text { Realizar encaminhamentos para rede de atenção } \\
\text { especializada (CAPSia) e se necessário para a rede } \\
\text { de assistência social do município. Em caso de } \\
\text { risco de vida para o paciente, o Conselho Tutelar e } \\
\text { Ministério Público poderão ser acionados. }\end{array}$ \\
\hline Passo 05 & Monitoramento & $\begin{array}{l}\text { Orientação para pacientes, familiares ou } \\
\text { responsáveis sobre os encaminhamentos, prestando } \\
\text { assessoria até alta hospitalar. }\end{array}$ \\
\hline
\end{tabular}

Quadro elaborado pelos autores. Santa Cruz do Sul, 2018.

Observa-se que a atuação dos profissionais de Serviço Social, em casos de Saúde Mental no Serviço de Emergência, baseia-se no pragmatismo, visando a construção de um fluxo de atendimento capaz de efetivar o acolhimento, planejamento, encaminhamentos e o monitoramento do caso. A execução deste fluxo de atendimento, seguindo os passos elencados, dependerá da complexidade da situação e tempo de permanência do paciente no Serviço de Emergência ou de sua adesão ao tratamento. Por ser uma unidade de saúde com uma constante demanda de leitos, é necessário planejar os encaminhamentos do paciente junto com a equipe médica e de psicologia do Serviço de Emergência, possibilitando soluções efetivas para os casos. Além disso, esses encaminhamentos devem servir como uma ferramenta de efetivação do tratamento, garantindo a imediata inclusão do paciente ao tratamento psiquiátrico, psicológico e social e prevenindo contra novas tentativas de suicídio ${ }^{3}$.

Durante a coleta de dados, foi possível identificar que os procedimentos de trabalho das Assistentes Sociais condizem com a efetivação do tratamento, seguindo passos importantes para adesão do paciente ao CAPSIA. Contudo, em muitos casos, deve-se

\footnotetext{
3 “A intervenção precoce depois de uma tentativa de suicídio é vital porque os primeiros meses são o período de maior risco para novas tentativas. No entanto, as taxas de adesão são baixas em indivíduos que tentam suicídio. Estima-se que até $60 \%$ dos indivíduos que tentaram suicídio não frequentam mais de uma semana de tratamento após alta do serviço de emergência." (Vidal \& Gontijo, 2013, pg.: 112).
} 
encaminhar o paciente para outros serviços e Políticas Públicas. O quadro a seguir representa a sistematização dos encaminhamentos realizados pelas profissionais entrevistadas.

Quadro 2. Encaminhamentos realizados pelo Serviço de Emergência em casos de tentativa de suicídio de adolescentes, elencados pelos profissionais entrevistados.

\begin{tabular}{|c|c|}
\hline Serviços de Referência & Formas de encaminhamento do Serviço Social \\
\hline CAPSIA & $\begin{array}{l}\text { Elaborado carta de encaminhamento, contendo informações } \\
\text { básicas do paciente, quadro clínico, motivo da internação e } \\
\text { condutas da equipe de saúde. Deve-se entregar a carta de } \\
\text { referência para um familiar ou responsável. Se necessário, o caso } \\
\text { deve ser ainda referenciado por contato telefônico e e-mail. }\end{array}$ \\
\hline CRAS/CREAS & $\begin{array}{l}\text { Enviado relatório via e-mail para a Secretaria Municipal de } \\
\text { Políticas Públicas solicitando acompanhamento familiar. }\end{array}$ \\
\hline Conselho Tutelar & $\begin{array}{l}\text { Comunicado via contato telefônico. Solicitado acompanhamento } \\
\text { do núcleo familiar. }\end{array}$ \\
\hline Ministério Público & Enviado ofício protocolado via e-mail. \\
\hline $\begin{array}{l}\text { Hospitais de Referência } \\
\text { Psiquiátrica }\end{array}$ & $\begin{array}{l}\text { O risco a vida ou a necessidade de internação é constatada pela } \\
\text { equipe médica, podendo o Serviço Social participar do } \\
\text { planejamento desta conduta. A referência hospitalar é realizada } \\
\text { através de documento específico, com assinatura do médico } \\
\text { plantonista responsável. }\end{array}$ \\
\hline
\end{tabular}

Quadro elaborado pelos autores. Santa Cruz do Sul, 2018.

Os encaminhamentos realizados pelo Serviço Social visam garantir a continuidade do tratamento e o envolvimento dos responsáveis no cuidado do paciente, criando um fluxo de referência e contra-referência ${ }^{4}$ com a rede de saúde municipal. O Centro de Atenção Psicossocial da Infância e Adolescência (CAPSIA) ${ }^{5}$ é o principal serviço regional para atendimento de quadros psiquiátricos para menores de 18 anos, sendo assim a principal referência para crianças e adolescentes atendidos no Serviço de Emergência do Hospital Santa Cruz por tentativa ou ideação suicida. O encaminhamento para o CAPSIA foi visto como essencial para as profissionais entrevistadas e se dá através de carta de encaminhamento, email ou via contato telefônico. Além do CAPSIA, outros serviços da rede de saúde podem ser

\footnotetext{
${ }^{4}$ Referência e contra-referência compõem um sistema organizador dos aparelhos de saúde, no sentido de permitir o acesso a todo o tipo de serviço oferecido pelo SUS. Nesse contexto, a atenção básica é considerada a "porta de entrada" do serviço de saúde, pois a depender da necessidade do usuário, o mesmo é referenciado para uma unidade com maior complexidade (unidade de referência). Assim, após o atendimento ou acompanhamento da unidade de referência, o usuário deve ser contra-referenciado para a atenção básica, para que o mesmo continue a ser assistido. (BRASIL, 2011).

${ }^{5}$ O CAPSIA é uma unidade de saúde vinculada à Secretaria Municipal de Saúde de Santa Cruz do Sul - RS e destina-se ao atendimento de crianças e adolescentes usuários de substâncias psicoativas e/ou que apresentem transtornos mentais graves e persistentes, que por sua condição psíquica estão levando a dificuldades de manter ou estabelecer laços sociais. A equipe técnica multiprofissional é composta por: 4 Psicólogas, 1 Fonoaudióloga, 2 Psiquiatras, 1 Terapeuta ocupacional, 1 enfermeira, 1 técnica de enfermagem, 1 Assistente Social, 1 Educador físico, 1 Redutor de danos e 1 Nutricionista. (Fonte: www.capsiascs.blogspot.com.br; acesso em: 02/05/2018).
} 
acionados, como o ESF de referência e NASF. A comunicação com escola, trabalho e demais espaços sociais ocupados pelo paciente, será acordado com o núcleo familiar, respeitando e analisando as particularidades de cada caso. O encaminhamento para as demais Políticas Públicas, como Assistência Social e Poder Judiciário, também dependerá das situações e dos riscos sociais do paciente.

Em situações de vulnerabilidade social do núcleo familiar, é necessário realizar encaminhamento para a rede de assistência social do município a fim de acompanhar e prestar suporte a família. O Centro de Referência da Assistência Social (CRAS) e o Centro de Referência Especializado da Assistência Social (CREAS) são os principais serviços disponibilizados pela Secretaria de Políticas Públicas de Santa Cruz do Sul. O Conselho Tutelar também poderá ser acionado sempre que se perceber negligência ou ausência de cuidado dos familiares ou nos casos de jovens em situação de abandono. Além destes, o Ministério Público é um dos equipamentos mais importantes na garantia de direitos de crianças e adolescentes e poderá ser acionado sempre que a equipe considerar necessário.

Destaca-se que, para dimensionar ao leitor a frequência de pacientes adolescentes atendidos no serviço de emergência por ideação suicida ou tentativa de suicídio, seria necessário apresentar dados quantitativos destes casos. Contudo, não obtivemos acesso ao número total de pacientes atendidos nos últimos anos, no município e no SE, específico para crianças e adolescentes com tentativa de suicídio. O Serviço de Emergência no qual esta pesquisa estrutura-se não é a única porta de entrada, tampouco a principal, para estes casos no município. O que podemos afirmar é a observação e avaliação empírica dos profissionais sobre o aumento dos casos de tentativa de suicídio entre os jovens, percebida tanto nas unidades de urgência e emergência, como no CAPSIA, Centro Materno Infantil (CEMAI) e Sistema Integrado de Saúde (SIS-UNISC), serviços que prestam assistência em situações de ideação suicida de crianças e adolescentes. De fato, no SE do HSC, são atendidas apenas os casos mais graves, como lesões autoprovocadas graves, intoxicação exógena e tentativas de suicídio que causem lesões e fraturas. A saber, no período de aplicação desta pesquisa, ouve o registro pessoal dos autores de 15 (quinze) casos de tentativa de suicídio de adolescentes no ano de 2018. Este número tende a ser maior, pois outros profissionais estão envolvidos nesta problemática, como psicólogos, enfermeiros, médicos emergencistas e psiquiatras, os quais mantém rotinas e fluxos de trabalho próprios. Além disso, estudos recentes apontam que apenas uma de cada três tentativas de suicídio são encaminhadas para uma unidade de saúde, sendo este um fenômeno velado a realidade dos dados (BOTEGA, 2014).

Embora, mesmo sem a qualificação de dados quantitativos que comprovem a 
incidência de tentativas de suicídio de adolescentes no serviço, é consenso na literatura contemporânea sobre o fenômeno e entre os profissionais de Saúde Mental do município o aumento do público jovem aos serviços de saúde, por demandas psicossociais. O CAPSIA registra entrada destes casos semanalmente, assim como o SIS-UNISC. As escolas municipais

e estaduais, da mesma forma, solicitam semanalmente suporte para acolhimento e atendimento de alunos com ideação suicida ou com ferimentos de automutilação. Neste contexto, se faz necessário a aplicação de pesquisa específica para verificar esta observação empírica, e fundamentar futuros estudos sobre o fenômeno do suicídio entre jovens, os quais não puderam ser abordados neste trabalho.

\section{Comunicação entre as equipes de saúde do Hospital Santa Cruz e Rede de Saúde Mental de Santa Cruz do Sul}

A partir dos dados coletados foi possível identificar a sistemática e efetividade da comunicação entre o Serviço de Emergência observado e a rede de atenção à Saúde Mental do município de Santa Cruz do Sul. Percebeu-se que a relação entre as equipes de saúde do Hospital e do CAPSIA é satisfatória, sendo constituída uma rede interna entre os profissionais de referência de cada serviço. Constatou-se que nos últimos cinco anos, ouve poucas mudanças no quadro de trabalhadores do SIAP e do CAPSIA, possibilitando uma relação entre os profissionais pautada na confiança e familiaridade entre as equipes. Outro fator levantado na pesquisa é a inserção dos residentes de Serviço Social no próprio CAPSIA, durante o primeiro ano de residência, no qual é realizado 384 horas no serviço, dando ao residente a oportunidade de conhecer e atuar na principal unidade de atendimento à Saúde Mental para crianças e adolescentes da região. As ex-residentes entrevistadas apontam este fator como determinante para qualificar a atuação profissional no Serviço de Emergência, sendo considerado por $100 \%$ das profissionais uma experiência valiosa na formação profissional.

Contudo, mesmo com a aproximação das equipes, foi alertado sobre a inconstância no processo de referência e contra-referência dos casos. Foi identificado na coleta de dados que este processo nem sempre acontece, o que dificulta o monitoramento do paciente em futuras internações. Considera-se que comunicar a equipe do Hospital sobre o acolhimento do paciente encaminhado ou informar sua inclusão no quadro de pacientes facilitaria no controle e conclusão dos casos atendidos no Serviço de Emergência.

Por fim, foi solicitado aos entrevistados um parecer sobre a formação ofertada no Programa de Residência Multiprofissional em Saúde, com ênfase em Urgência, Emergência e 
Terapia Intensiva, a fim de contribuir com a elaboração deste trabalho. As ex-residentes de Serviço Social do PRMS enfatizaram a importância do programa para a formação profissional em saúde, destacando a relação com as outras áreas da saúde e como a oportunidade de atuar em conjunto possibilitou enxergar os pacientes de forma biopsicossocial. Evidenciaram em suas respostas a importância do Serviço Social para a manutenção da saúde, considerando aspectos socioassistenciais como primordiais para o cuidado e de como as outras áreas da saúde puderam enxergar a atuação do Assistente Social nos casos clínicos. Os rounds multiprofissionais ${ }^{6}$ foram apontados como um espaço importante para o diálogo entre os diferentes saberes e ouve críticas quanto ao plano pedagógico do PRMS, em especial a escassa oferta de disciplinas do programa ${ }^{7}$.

${ }^{6}$ Os Rounds Multiprofissionais são realizados semanalmente como atividade pedagógica do Programa de Residência Multiprofissional em Saúde do Hospital Santa Cruz, tendo no mínimo um residente de cada área do Programa, onde se discute casos emergentes e/ou clinicamente complexos. Esses momentos de discussão contam sempre com a participação de um profissional preceptor.

${ }^{7}$ Atualmente o PRMS conta com um plano pedagógico aprovado anualmente em reunião de colegiado, onde consta na grade curricular do programa as disciplinas de "Bioestatística", "Pesquisa em Saúde I", "Pesquisa em Saúde II", "Aulas de Tópicos", "Núcleo Flexível” (atividades extracurriculares), tutorias e preceptorias. Além das disciplinas, existem as atividades quinzenais e semanais como o "Clube de Revistas" (onde os residentes apresentam artigos científicos) e o "Grand Round" (Espaço de apresentação de casos clínicos). 


\section{DISCUSSÃO}

Observou-se que os procedimentos de trabalho desencadeados pelos residentes de Serviço Social em casos de tentativa ou ideação suicida de adolescentes, atendidos em um Serviço de Emergência, condizem com a literatura vigente da categoria profissional. Segundo Mioto \& Nogueira (2009), a atuação profissional em saúde baseia-se em três pilares: necessidades sociais em saúde; direito à saúde e produção da saúde.

As necessidades sociais em saúde são historicamente construídas e determinadas
pelo movimento societário. O direito à saúde, mediado pelas políticas públicas, as
quais refletem um patamar determinado da relação Estado e Sociedade, é
operacionalizado através dos sistemas e serviços de saúde, envolvendo a gestão, o
planejamento e a avaliação, além do controle social. A produção de saúde é
entendida como um processo que se articula a partir das transformaçóes econômicas,
sociais e políticas, das ações de vigilância à saúde e das práticas de assistência à
saúde. (MIOTO \& NOGUEIRA, 2009, p. 223).

O desafio de vincular a categoria teórico-metodológica com o projeto ético-político da profissão, visando a universalidade no atendimento e a participação popular, se intensifica ao pensar a atuação do Assistente Social na Saúde. A especialidade de Urgência e Emergência traz novos desafios aos profissionais, devido à complexidade das demandas e a necessidade de respostas ágeis, tanto para os pacientes quanto para as equipes de saúde. Essa condição emergencista do trabalho centraliza a atuação do Assistente Social exclusivamente na ação/tarefa que executa, reforçando estereótipos de subalternidade da profissão. A ação profissional $^{8}$ em casos de tentativa ou ideação suicida de adolescentes foi apresentada sem divergências pelos dados coletados na pesquisa. Possibilitou-se, inclusive, a construção de um protocolo, dividido em passos de intervenção, sendo em ordem: Acolhimento/ Atendimento, Levantamento de informações, Planejamento, Encaminhamento e Monitoramento.

Consideramos relevante avaliar se as convergências de informações coletadas com este estudo apontam para uma prática reflexiva e estruturada entre as profissionais ou se de fato evidencia o pragmatismo exigido aos Assistentes Sociais pelos serviços de saúde. Compreendendo que a amostra limita esta análise, colocamos o questionamento para futuros estudos.

De fato, as intervenções necessárias em casos de Saúde Mental e a complexidade em

\footnotetext{
8 A ação profissional define-se como um conjunto de procedimentos, atos, atividades pertinentes a uma determinada profissão e realizadas por sujeitos/ profissionais de forma responsável, consciente. Portanto, contém tanto uma dimensão operativa quanto uma dimensão ética, e expressa no momento em que se realiza o processo de apropriação que os profissionais fazem dos fundamentos teórico-metodológicos e ético-políticos da profissão em determinado momento histórico. São as ações profissionais que colocam em movimento, no âmbito da realidade social, determinados projetos de profissão. Estes, por sua vez, implicam em diferentes concepções de homem, de sociedade e de relações sociais (MIOTO, 2001).
} 
atuar com adolescentes exigem do profissional não apenas a competência prática-operativa, mas a reflexão constante de suas intervenções, tornando o seu trabalho em uma prática reflexiva. Para Guerra (2000) "o processo de trabalho é compreendido como um conjunto de atividades prático-reflexivas voltadas para o alcance de finalidades, as quais dependem da existência, da adequação e da criação dos meios e das condições objetivas e subjetivas" (p. 03). Entende-se que é necessário analisar diversos fatores relevantes ao trabalho do Assistente Social, como o espaço sócio-ocupacional, serviços disponíveis rede socioassistencial e de saúde, relações e correlações de forças entre os profissionais, entidade, serviços e gestores, além do arsenal teórico-metodológico referente ao instrumental do Serviço Social. Pensar a prática-reflexiva, é analisar todos esses elementos e mais, a fim de garantir a qualificação constante do atendimento aos pacientes.

O estudo constatou ainda a sistemática no processo de encaminhamento e comunicação entre as equipes de referência dos serviços de Saúde Mental, em especial o CAPSIA e a equipe de saúde do Serviço de Emergência. Essa comunicação, seja para discutir casos ou para encaminhar pacientes, se faz de diversas formas, como: encaminhamento médico; encaminhamento Psicossocial, documento de referência e contra-referência, carta de encaminhamento, correio eletrônico, ofício e contato telefônico. Não ouve relatos de discussões de casos entre as equipes, fator que poderia servir como aliado ao tratamento pósalta hospitalar. Também não surgiu nos relatos qualquer experiência de atendimento conjunto entre as equipes do CAPSIA e Serviço de Emergência. Contudo, nota-se que as profissionais entrevistadas referiam boa comunicação entre os serviços, devido a pouca rotatividade de profissionais e confiança no trabalho mútuo. Türck (2002) discursa sobre isso ao apresentar a Rede Interna" e de como esta rede passa "pelo compartilhamento dos sujeitos, pela intercomunicação entre eles, pela inclusão e pelo interesse comum” (p. 31). Para Türck (2002) o trabalho de rede deve ser observado a partir dos sujeitos envolvidos com igual importância às entidades que fazem parte desta rede.

$\mathrm{Na}$ Rede Interna, por exemplo, é necessário que os sujeitos envolvidos “interajam entre si e se disponibilizem a compartilhar afeto e conhecimento, pressuposto fundamental para qualquer ação que implique a construção coletiva da solidariedade" (TÜRCK, 2002, p. 27).

9 "Síntese da experiência e conhecimento profissional dos atores sociais em uma ação conjunta e participativa, objetivando a construção de uma práxis interdisciplinar no atendimento a situações individuais e/ou coletivas que emergem em um determinado contexto social." (TÜRCK, 2002, p. 03). Trata-se dos indivíduos que compõe uma determinada rede de proteção e/ou garantia de direitos, entendendo que seus conhecimentos e características pessoais interagem com o cotidiano do trabalho pretendido. 
A formação das Redes Internas passa pelo compartilhamento dos sujeitos, pela intercomunicação entre eles, pela inclusão e pelo interesse comum. $O$ interesse comum na resolução de qualquer situação, seja em relação a pessoas, seja na execução de tarefas, necessita de um processo de solidariedade na construção coletiva de uma resposta. (TÜRCK, 2002, p. 31).

Para Türck (2002) as pessoas/profissionais que compõem essas Redes são capazes de influenciar diretamente no próprio funcionamento da rede, por questões individuais, como o personalismo, a disputa, o conformismo. Da mesma forma, estes profissionais, ao incorporarem o trabalho de rede ao seu cotidiano profissional, são capazes de agilizar e qualificar o trabalho.

Portanto, observa-se que a relação da categoria teórico-metológica e o projeto éticopolítico do Assistente Social, a prática-reflexiva e a relação da Rede Interna entre os serviços de saúde, estão intrinsecamente relacionadas ao atendimento integral de adolescentes que tentaram ou planejam o suicídio. As possibilidades de intervenção do Serviço Social nesta demanda perpassam todo o conjunto de ações voltadas ao cuidado, tratamento e prevenção. Este profissional, quando introduzido em uma equipe de um Serviço de Emergência, deve, além de sua atuação profissional direta, manter o posicionamento crítico quanto aos processos de trabalho instituídos. O Assistente Social tem um compromisso ético e político com os direitos de seus pacientes, devendo "decifrar a realidade e construir propostas de trabalho criativas e capazes de preservar e efetivar a partir de demandas emergentes no cotidiano. Enfim ser um profissional propositivo não só executivo” (IAMAMOTO, 2005, p.20). 


\section{CONCLUSÃO}

Com o objetivo de analisar os processos de trabalho dos Assistentes Sociais e residentes em casos de tentativa ou ideação suicida de adolescentes, observou-se que a atuação destes profissionais qualifica e estreita a relação entre o Serviço de Emergência e a Rede de Saúde Mental do município de Santa Cruz do Sul. Com a inclusão do Serviço Social na equipe multiprofissional do SE os casos de tentativa de suicídio passaram a ser avaliados com maior abrangência, abordando questões clínicas, psicológicas e sociais dos pacientes. Os procedimentos e intervenções realizadas pelos Assistentes Sociais ampliam as possibilidades de cuidado, resgatando informações importantes para o tratamento do paciente e sua inclusão nos equipamentos de saúde especializados. Suas competências profissionais contribuem para o aprimoramento das equipes e qualificação dos fluxos de trabalho, efetivando a atenção integral dos usuários.

O Assistente Social dispõe de um arsenal teórico-prático capaz de atuar em expressões da questão social e em diferentes demandas da saúde. Em casos de tentativa de suicídio de adolescentes é possível resgatar, logo nos primeiros contatos, informações sobre a rede familiar e de apoio, vulnerabilidade social, vinculação com a escola, condições de moradia, entre outros fatores importantes para compreender o cenário social do paciente. A intervenção do Assistente Social deve focar no cuidado e nas possibilidades de apoio em seu núcleo familiar. Suas técnicas devem auxiliar no manejo deste jovem, vinculando-o com a equipe do Serviço de Emergência ou preparando-o para o acolhimento em outros serviços, como o CAPSIA, por exemplo. Além disso, este profissional é capaz de identificar e intervir em situações de violência, abandono, negligência, abuso, maus tratos, bullying, exploração, ou qualquer violação de direito sofrida pelo paciente, que são as principais causas de tentativas de suicídio na adolescência.

Constatou-se que em um Serviço de Emergência, estas intervenções podem ser dificultadas pelo constante fluxo de pacientes e características emergencistas da unidade de saúde. O Assistente Social que atuar em equipamentos de Urgência e Emergência deve instruir a equipe multiprofissional para que o seu trabalho seja compreendido pelos demais trabalhadores. Esta compreensão foi vista como essencial para que as intervenções não sejam prejudicadas pelos protocolos ou rotinas da unidade, construindo referência com a equipe e garantindo condições de um atendimento qualificado, ético e sigiloso. Alguns procedimentos de trabalho do Assistente Social necessitam de um tempo maior de intervenção, como o resgate de informações, organização familiar e contato com serviços da rede socioassistencial, de saúde e de proteção. Para isso, é necessário que o SE estabeleça rotinas, fluxos ou 
protocolos que permitam a atuação do Assistente Social de forma que não fira o Código de Ética Profissional. A necessidade de um espaço adequado para atendimento também foi trazido como fundamental, porém, muitos Serviços de Emergência não dispõem de espaço físico para que o profissional execute seus atendimentos. Para isso, é necessário organizar com a própria equipe a criação deste espaço, seja utilizando salas apropriadas ou criando mecanismos que preservem o sigilo profissional.

Em situações graves, como a tentativa de suicídio de adolescentes, pode-se perceber, a partir dos relatos das profissionais entrevistadas, que existe certo despreparo dos demais profissionais para atender esta demanda. A própria questão do suicídio ainda é vista com preconceito, estigmas e incertezas para as equipes de saúde. O Assistente Social, em sua atuação profissional, deve atentar-se para as condutas dos demais trabalhadores, evitando que a falta de informação dos profissionais de saúde prejudique o manejo ou a adesão do paciente ao tratamento. Esta tarefa foi apresentada como complicadora na atuação do Serviço Social em um Serviço de Emergência, diante da constante necessidade de conquista por espaço profissional.

Diante dos dados, pode-se perceber que o Programa de Residência Multiprofissional em Saúde contribuiu significativamente para que o Serviço Social conquistasse este espaço junto ao Serviço de Emergência. A ênfase de Urgência, Emergência e Terapia Intensiva traz novos desafios aos Assistentes Sociais, evidenciando necessidades dos pacientes, que antes não eram percebidas em uma unidade de saúde de emergência. Considerando que o PRMS é recente no Hospital Santa Cruz (iniciado em 2014), é possível afirmar que importantes avanços foram conquistados pela atuação das residentes de Serviço Social. A atuação do Assistente Social no Serviço de Emergência criou referência para as equipes, ampliando as possibilidades de intervenção deste profissional e qualificando a comunicação com os demais serviços de saúde e de assistência social do município.

Contudo, apesar de contemplar 100\% das Assistentes Sociais que atuaram no Serviço de Emergência do Hospital Santa Cruz, desde a implantação da RAPS em 2011, este trabalho não dialoga com outras pesquisas referentes aos processos de trabalho do Serviço Social na saúde. Falar sobre a atuação do Assistente Social - diante da constante investida de ordem conservadora nas Políticas Públicas e considerando o aumento da desigualdade social no país - é uma ação necessária. O Assistente Social deve se manter vigilante, independente da Política Pública que atue, reivindicando espaços de trabalho e construindo estratégias de enfrentamento às expressões da questão social.

Por fim, salienta-se a necessidade de aprofundar estes estudos, analisando a atuação de 
outros Assistentes Sociais que desempenham seu trabalho em unidades de Urgência e Emergência, e destacando as estratégias que estes profissionais encontraram para exercer seus processos de trabalho. Apesar da pequena amostragem desta pesquisa, seus resultados serviram para fundamentar os procedimentos de trabalho adotados pelo Serviço Social dentro do Serviço de Emergência do Hospital Santa Cruz. As práticas profissionais apresentadas foram consideradas como essenciais para o atendimento e acompanhamento de casos de tentativa de suicídio e ideação suicida de adolescentes.

\title{
THE SOCIAL SERVICE IN THE CARE OF PSYCHIATRIC EMERGENCIES: Work PROCESSES OF SOCIAL WORKERS AND RESIDENTS IN THE CARE OF ADOLESCENTS WITH SUICIDAL IDEATION AND ATTEMPETD SUICIDE
}

\begin{abstract}
This work presents reflections on the phenomenon of suicide in adolescence, from the analysis of work processes of social workers, residents of an Emergency Service of SUS. This study arose the need to qualify work methodologies are consistent with the professional and ethical principles of Social Work and Mental Health. It is a descriptive study with a qualitative approach, consisting of six social workers, being three former residents of Multiprofessional Residency Program in health with emphasis on intensive, Urgency and Emergency and three Social Workers of the Santa Cruz Hospital. The data collection was performed through interviews between the months of March and June 2018. It was discovered that $83 \%$ of professionals made visits to the emergency room and $67 \%$ underwent direct intervention with adolescents with suicidal ideation or attempt. It revealed that the flow of treatment of these cases as the host, planning, referrals and monitoring the patient, assigning the qualification of customer service professional improvement due to the Multiprofessional Residency Program in Health.
\end{abstract}

Keywords: Suicide; Social Services; Adolescence; Emergency Service.

\section{EL SERVICIO SOCIAL EN LA ATENCIÓN DE EMERGENCIAS PSIQUIÁTRICAS: PROCESOS DE TRABAJO DE ASISTENTES SOCIALES Y RESIDENTES EN LA ATENCIÓN DE PACIENTES ADOLESCENTES CON IDEACIÓN E INTENTO DE SUICIDIO}

\section{RESUMEN}

Este trabajo presenta reflexiones sobre el fenómeno del suicidio en la adolescencia, a partir del análisis de los procesos de trabajo de Asistentes Sociales residentes de un Servicio de Emergencia del SUS. Este estudio surge por la necesidad de calificar metodologías de trabajo acorde con los principios profesionales y éticos del Servicio Social y Salud Mental. Se trata de un estudio descriptivo con abordaje cualitativo, constituido por seis asistentes sociales, siendo tres ex residentes del Programa de Residencia Multiprofesional en Salud con énfasis en Intensivismo, Urgencia y Emergencia y tres Asistentes Sociales del Hospital Santa Cruz. La recolección de datos fue realizada a través de una entrevista semiestructurada entre los meses 
de marzo y junio de 2018. Se descubrió que el $83 \%$ de las profesionales realizaron atendimientos en el Servicio de Emergencia y el 67\% realizaron intervenciones directas con adolescentes con intento o ideación suicida. Se desveló el flujo de atención de estos casos como la acogida, planificación, encaminamientos y el monitoreo del paciente, atribuyendo la calificación de la atención al perfeccionamiento profesional resultante del Programa de Residencia Multiprofesional en Salud.

Palabras clave: Suicidio; Servicio Social; la adolescencia; Servicio de Emergencia. 


\section{REFERÊNCIAS}

AVANCI, Rita de Cássia. PEDRÃO, Luiz Jorge. COSTA JÚNIOR, Moacyr Lobo da. Perfil do adolescente que tenta suicídio em uma unidade de emergência. Revista Brasileira de $\begin{array}{llllll}\text { Enfermagem } & \text { [en linea] 2005, } 58 \text { (Sep-Oct): Disponível }\end{array}$ em:<http://www.redalyc.org/articulo.oa?id=267019626007> Acesso em: 02/05/2018.

BERTOlOTE, J. M., \& FlEISCHMANN, A. (2004). Suicídio e doença mental: uma perspectiva global. In N. J. Botega \& B. G. Werlang (Orgs.), Comportamento suicida. Porto Alegre: ArtMed, p. 35-44

BOTEGA, Neury José. Comportamento suicida: epidemiologia. Psicol. USP, São Paulo , v. 25, n. 3, p. 231-236, dez. 2014. Doi: 10.1590/0103-6564D20140004.

BRASIL. Constituição Federal de 1988. Direitos Sociais e Lei nº 8.662, de 07 de junho de1993. Brasília, DF, jun/dez 2000. In: Coletânea de Leis e Resoluções. Assistente Social: ética e direitos. 4. ed. Rio de Janeiro: CRESS, 2004.

Lei 8.069/90. Estatuto da Criança e do Adolescente. Brasília, 1990. In: Conselho Regional de Serviço Social - CRESS $10^{a}$ Região. Coletânea de leis: revista e ampliada. Porto Alegre: CRESS, 2005.

Ministério do Desenvolvimento Social e Combate à Fome. Plano Nacional de Promoção, Proteção e Defesa do Direito de Crianças e Adolescentes à Convivência Familiar e Comunitária. Presidência da República. Secretaria Especial dos Direitos Humanos. MDS: Brasília, 2006.

. Ministério da Saúde. Portaria n ${ }^{\circ} 3.088$, de 23 de dezembro de 2011. Institui a Rede de Atenção Psicossocial para pessoas com sofrimento ou transtorno mental e com necessidades decorrentes do uso de crack, álcool e outras drogas, no âmbito do Sistema Único de Saúde. Brasília, DF: Ministério da Saúde, 2011. Disponível em: <http://www.brasilsus.com.br/legislacoes/gm/111276-3088.html > . Acesso em: 27 abr. 2018.

CARVALHO, Raul de. IAMAMOTO, Marilda Villela. Relações Sociais e Serviço Social no Brasil: Esboço de uma interpretação histórico-metodológica. 20. ed. São Paulo: Cortez, CELATS, 2007.

COSTA, M. D. H. O trabalho nos serviços de saúde e a inserção dos(as) assistentes sociais. Serviço Social e Sociedade. n. 62. São Paulo: Cortez, 2000.

DE LEO, D., BERTOLOTE, J., \& LESTER, D. La violencia autoinfligida. In E. G. Krug, L. L. Dahlberg, J. A. Mercy, A. B. Zuvi, \& P. R. Lozano (Orgs.), Informe Mundial de la violencia e de la salud. Washington: Organización Panamericana de la Salud. 2003.p. 200-231

DUTRA, E. Comportamentos autodestrutivos em crianças e adolescentes: Orientações que podem ajudar a identificar e prevenir. In: C.S. HUTZ (Ed.), Situações de risco e vulnerabilidade na infância e adolescência: Aspectos teóricos e estratégias de intervenção. Porto Alegre, Casa do Psicólogo. 2002, p. 53-87.

FALEIROS, Vicente de Paula. Alternativas Metodológicas da Pesquisa em Serviço Social. Serviço Social \& Sociedade. n. 21. São Paulo: Cortez, 1986, p. 05 - 17.

FERREIRA, José Wesley. Questão Social: apreensão e intervenção no trabalho dos Assistentes Sociais. Dissertação (mestrado). Porto Alegre: PUCRS, 2008.

GUERRA, Yolanda. Instrumentalidade do processo de trabalho e Serviço Social. In: Revista Serviço Social e Sociedade n. 62. São Paulo: Cortez, 2000. 
IAMAMOTO, Marilda Vilela. O Serviço Social na Contemporaneidade: trabalho e formação profissional. 9 ed. São Paulo, Cortez, 2005.

MARTINELLI, Maria Lúcia. O trabalho do assistente social em contextos hospitalares: desafios cotidianos. Serv. Soc. Soc. [online] n.107. 2011, p.497-508. Disponível em: <http://www.scielo.br/pdf/sssoc/n107/07.pdf>. Acesso em: 22/07/2018.

MINAYO, Maria Cecília de Souza. Pesquisa Social: teoria, método e criatividade. 22. ed. São Paulo: Vozes, 2003.

MIOTO, Regina Célia Tamaso. NOGUEIRA, Vera Maria Ribeiro. SER Social, Brasília, v. 11, n. 25, jul./dez. 2009, p. 221-243.

MIOTO, Regina Célia Tamaso. Processo de construção do espaço profissional do assistente social em contexto multiprofissional:um estudo sobre o Serviço Social na Estratégia Saúde da Família. Projeto de Pesquisa: UFSC/CNPq. Florianópolis, 2004.

MIOTO, Regina Célia Tamaso. Perícia Social: proposta de um percurso operativo. Revista Serviço Social \& Sociedade. n. 67. São Paulo: Cortez, 2001, p. 145-158

MIOTO, Regina Célia Tamaso. Família e Serviço Social: contribuições para o debate. In: Serviço Social \& Sociedade, nº55. São Paulo: Cortez, 1997.

NOGUEIRA, V. M. R.; PIRES, D. E. P. Direito à saúde - convite à reflexão. Cadernos de Saúde Pública. Rio de Janeiro: 2004. v. 20, n. 3, p. 753-760

PEREIRA, Marília do Nascimento. A superexposição de crianças e adolescentes nas redes sociais: necessária cautela no uso das novas tecnologias para a formação de identidade. In: Congresso Internaconal de Direito e Contemporaneidade. $3^{\mathrm{a}}$ ed. 2015. Santa Maria / RS. Anais. Universidade Federal de Santa Maria, 2015. Disponível em: <http://coral.ufsm.br/congressodireito/anais/2015/6-14.pdf>. Acesso em: 04/05/2018.

TÜRCK, Maria da Graça Maurer Gomes. Rede Interna e Rede Social: O desafio permanente na teia das relações sociais. Coleção Amencar. Porto Alegre. Tomo Editorial. 2002.

VIDAL, C. E. L., \& GONTIJO, E. D. (2013). Tentativas de suicídio e o acolhimento nos serviços de urgência: a percepção de quem tenta. Cadernos Saúde Coletiva, 21(2), p. 108-114.

VIDAL, C. E. L., GONTIJO, E. C. D. M., \& Lima, L. A. (2013). Tentativas de suicídio: fatores prognósticos e estimativa do excesso de mortalidade. Cadernos de Saúde Pública, 29(1), p. $175-187$

\section{Sobre os autores}

Carlos Stavizki Junior é mestrando no Programa de Pós-Graduação em Desenvolvimento Regional (Mestrado e Doutorado) - PPGDR/UNISC (2019 - atual); Especialista em Atenção de Urgência e Emergência, realizado no Programa de Pós-graduação em Residência Multiprofissional em Saúde - Ênfase em Urgência, Emergência e Terapia Intensiva - APESC/ Hospital Santa Cruz (2017- 2019); Bacharel em Serviço Social pela Universidade de Santa Cruz do Sul - UNISC (2017); Bacharel em Teologia pelo Centro de Ensino Superior de Maringá - CESUMAR (2013); Membro do Comitê Municipal de Prevenção ao Suicídio de Santa Cruz do Sul - CMPS (desde 2017); Membro do Grupo de Pesquisa e Estudos Urbanos e Regionais - GEPEUR (desde 2019); Membro do Grupo de Estudos sobre Democracia e Políticas Públicas - GEDEPP (desde 2019); Bolsista CAPES Modalidade 1 - Mestrado. Endereço eletrônico: carlos_stavizki@hotmail.com 
do Vale do Rio do Peixe (1981), mestrado em Serviço Social pela Pontifícia Universidade Católica do Rio Grande do Sul (2001) e doutorado em Serviço Social pela Pontifícia Universidade Católica do Rio Grande do Sul (2008). Professora do Curso de Serviço Social da Universidade de Santa Cruz do Sul. Endereço eletrônico: eviccari@unisc.br 